\title{
Group of continuous transformations of real interval preserving tails of $G_{2}$-representation of numbers
}

\author{
M. V. Pratsiovytyi, I. M. Lysenko, and Yu. P. Maslova
}

\author{
Communicated by A. P. Petravchuk \\ Dedicated to the 70th anniversary \\ of Leonid Andriyovych Kurdachenko
}

\begin{abstract}
A BSTRACT. In the paper, we consider a two-symbol system of encoding for real numbers with two bases having different signs $g_{0}<1$ and $g_{1}=g_{0}-1$. Transformations (bijections of the set to itself) of interval $\left[0, g_{0}\right]$ preserving tails of this representation of numbers are studied. We prove constructively that the set of all continuous transformations from this class with respect to composition of functions forms an infinite non-abelian group such that increasing transformations form its proper subgroup. This group is a proper subgroup of the group of transformations preserving frequencies of digits of representations of numbers.
\end{abstract}

\section{Introduction}

Many two-symbol systems of encoding (representation) of fractional part of real numbers are known. They use the alphabet $A=\{0,1\}$ and are based on expansions of numbers in series, infinite products, continued fractions, etc. These systems identify a number as a sequence of zeros and

2010 MSC: 11H71, 26A46, 93B17.

Key words and phrases: two-symbol system of encoding for real numbers with two bases having different signs $\left(G_{2}\right.$-representation), tail of representation of number, continuous transformation of interval, left and right shift operators, continuous transformation preserving tails of representations. 
ones, i.e., as element of space $L=A \times A \times \ldots$ of sequences of zeros and ones. Every such a system has some advantages and restrictednesses as well as certain conveniences for solving some problems of number-theoretical, topological-metric and probabilistic kind. A system with two positive bases $\left(q_{0} \in(0,1), q_{1}=1-q_{0}\right)$ is among them. It generalizes classic binary system, has a self-similar geometry and zero redundancy. This is a system of $Q_{2}$-representation of numbers based on representation of a number by the series

$$
x=\alpha_{1} q_{1-\alpha_{1}}+\sum_{k=2}^{\infty}\left(\alpha_{k} q_{1-\alpha_{k}} \prod_{j=1}^{k-1} q_{\alpha_{j}}\right) \equiv \Delta_{\alpha_{1} \alpha_{2} \ldots \alpha_{k} \ldots}^{Q_{2}} .
$$

For $q_{0}=0,5$, it is a classic binary system.

In expression $\prod_{j=1}^{k} q_{\alpha_{j}}=q_{1}^{N_{0}(x, k)} q_{1}^{N_{1}(x, k)}$, where $N_{1}(x, k)=\alpha_{1}+\alpha_{2}+$ $\ldots+\alpha_{k}$ and $N_{0}(x, k)=k-N_{1}(x, k)$, one can see that this is a system with two bases. This system of encoding of numbers has various applications in metric and probabilistic theory of numbers, function theory and measure theory, fractal analysis and fractal geometry. For this system, the left shift operator is a discontinuous function, linear and increasing on cylinders of $\operatorname{rank} 1: \Delta_{0}^{Q_{2}}=\left[0, q_{0}\right]$ and $\Delta_{1}^{Q_{2}}=\left[q_{0}, 1\right]$.

In the papers [7], [8], an analogue of $Q_{2}$-representation of numbers, namely, system of encoding of numbers in interval $\left[0, g_{0}\right]$ with bases $g_{0} \in$ $(0,1)$ and $g_{1}=g_{0}-1$ having different signs is introduced. It is based on expansion of a number in the series

$$
x=\alpha_{1} g_{1-\alpha_{1}}+\sum_{k=2}^{\infty}\left(\alpha_{k} g_{1-\alpha_{k}} \prod_{j=1}^{k-1} g_{\alpha_{j}}\right) \equiv \Delta_{\alpha_{1} \alpha_{2} \ldots \alpha_{k} \ldots}^{G_{2}} .
$$

This system also has a zero redundancy (any number has at most two representations, and set of numbers having two representations is countable).

Generally speaking, series (1) has positive as well as negative terms. So $G_{2}$-representation is not topologically equivalent to $Q_{2}$-representation and it is not a simple reencoding of $Q_{2}$-representation. At the same time metric theories of these representations are similar. A vivid peculiarity of $G_{2}$-representation is the fact that left shift operator of $G_{2}$-representation is a continuous function on a whole interval $\left[0, g_{0}\right]$. This is a fundamental difference between this system and previously studied systems.

Real numbers in interval $\left[0, g_{0}\right]$ having two $G_{2}$-representations are called $G_{2}$-binary numbers, and numbers having a unique representation 
are called $G_{2^{-}}$unary numbers. It is known [10] that the set $B$ of all $G_{2^{-}}$ binary numbers is countable and consists of numbers with the following $G_{2}$-representation: $\Delta_{c_{1} \ldots c_{m} 01(0)}^{G_{2}}=\Delta_{c_{1} \ldots c_{m} 11(0)}^{G_{2}}$.

Algorithm for comparison of numbers in terms of their $G_{2}$-representations is the following.

Theorem 1 ([10]). Numbers $\Delta_{c_{1} c_{2} \ldots c_{m} 1 d_{1} d_{2} \ldots}^{G_{2}}=x_{1} \neq x_{2}=\Delta_{c_{1} c_{2} \ldots c_{m} 0 d_{1}^{\prime} d_{2}^{\prime} \ldots}^{G_{2}}$ are in the relation

$$
\begin{gathered}
x_{1} \geqslant x_{2} \quad \text { if } \quad \sigma_{m} \equiv c_{1}+c_{2}+\ldots+c_{m}=2 k, \\
x_{1} \leqslant x_{2} \quad \text { if } \quad \sigma_{m} \equiv c_{1}+c_{2}+\ldots+c_{m}=2 k-1 .
\end{gathered}
$$

In this paper, we consider continuous transformations of interval $\left[0, g_{0}\right]$, i.e., bijections of interval to itself, preserving "tails of $G_{2}$-representation of numbers", namely, we study group properties of the family of these transformations. Groups of transformations preserving tails of different representations of numbers were studied in the papers [2], [3], [4]. Specific properties of $G_{2}$-representation generate a peculiar corresponding transformation group having a non-trivial subgroup of increasing functions unlike other systems.

\section{Tails and tail sets}

Definition 1. We say that $G_{2}$-representations of numbers $x=\Delta_{\alpha_{1} \ldots \alpha_{n} \ldots}^{G_{2}}$ and $y=\Delta_{\beta_{1} \beta_{2} \ldots \beta_{n} \ldots}^{G_{2}}$ have the same tail if there exist positive integers $k$ and $m$ such that

$$
\alpha_{k+j}=\beta_{m+j}
$$

for any $j \in N$. We denote it symbolically by $x \sim y$.

Definition 2. Suppose that $k$ and $m$ are the smallest numbers satisfying condition (2). Then $G_{2}$-representation and corresponding number

$$
z \equiv[x \wedge y]=\Delta_{\alpha_{k+1} \alpha_{k+2} \ldots}^{G_{2}}=\Delta_{\beta_{m+1} \beta_{m+2} \ldots}^{G_{2}}
$$

is called a common tail of representations of numbers $x$ and $y$.

It is evident that binary relation $\sim$ ("has the same tail") is an equivalence relation, i.e., it is reflexive, symmetric and transitive. Thus it provides a partition of the set $Z$ of all $G_{2}$-representations of numbers in interval $\left[0, g_{0}\right]$ into the equivalence classes, and they form together a quotient set $W=Z / \sim$. Any element of the set $W$ is called a tail set, this set is uniquely determined by an arbitrary its element. 
Theorem 2. Any tail set is a countable everywhere dense in interval $\left[0, g_{0}\right]$ set. The set $W$ of all tail sets is a continuum set.

Proof. Suppose $K_{x}$ is a tail set containing the number $x, K_{1}$ is a set of all numbers $y$ such that $[y \wedge x]=x$, and $K_{n}$ is a set of all numbers $y$ such that $\left[y \wedge \Delta_{\alpha_{n+1}(x) \alpha_{n+2}(x) \ldots}^{G_{2}}\right]=\Delta_{\alpha_{n+1}(x) \alpha_{n+2}(x) \ldots}^{G_{2}}$.

It is evident that every set $K_{n}(n \in N)$ is countable and $K_{x}=\bigcup_{n} K_{n}$. Thus $K_{x}$ is a countable set because it is a countable union of countable sets.

An arbitrary cylinder $\Delta_{c_{1} \ldots c_{n}}^{G_{2}}$ contains points of tail set $K_{x}$ because points $\Delta_{c_{1} \ldots c_{n} \alpha_{1}(x) \alpha_{2}(x) \ldots,}^{G_{2}} \Delta_{c_{1} \ldots c_{n} \alpha_{2}(x) \alpha_{3}(x) \ldots}^{G_{2} \ldots c_{n}}$, and $\Delta_{c_{1} \ldots c_{n} \alpha_{k}(x) \alpha_{k+1}(x) \ldots}^{G_{2}}$ belong to this cylinder. This proves that the set $K_{x}$ is everywhere dense in interval $\left[0, g_{0}\right]$.

The set $W$ is a continuum set. Indeed, suppose that it is a countable set. Then we see that interval $\left[0, g_{0}\right]$ is a countable set because it is a countable union of countable sets. This contradicts the fact that interval is a continuum set.

Note that all $G_{2}$-binary numbers belong to the same tail set.

\section{Left and right shift operators of $G_{2}$-representation of numbers}

Theorem 3 ([8]). Left shift operator $\omega$ of $G_{2}$-representation of numbers in interval $\left[0, g_{0}\right]$ defined by equality

$$
\omega\left(\Delta_{\alpha_{1} \alpha_{2} \ldots \alpha_{n} \ldots}^{G_{2}}\right)=\Delta_{\alpha_{2} \alpha_{3} \ldots \alpha_{n} \ldots}^{G_{2}}
$$

in space of $G_{2}$-representations, is analytically expressed in the form $\omega(x)=\frac{1}{g_{\alpha_{1}(x)}} x-\frac{\delta_{\alpha_{1}(x)}}{g_{\alpha_{1}(x)}}$, is a continuous well-defined function on $\left[0, g_{0}\right]$, is linear on every cylinder of rank 1 , is increasing on $\Delta_{0}$ and decreasing on $\Delta_{1}$.

Numbers

$$
0=\Delta_{(0)}^{G_{2}} \quad \text { and } \quad \Delta_{(1)}^{G_{2}}=g_{0}+g_{0} g_{1}+g_{0} g_{1}^{2}+\ldots=\frac{g_{0}}{2+g_{0}}
$$

are invariant points of left shift operator $\omega$.

Remark 1. The last theorem shows an essential difference between $G_{2^{-}}$ representation and other known two-symbol representations, in particular, $Q_{2}^{*}$-representation, $\tilde{Q}$-representation [9] and $A_{2}$-continued fractions [3]. 
Let $n$ be a positive integer that is greater than 1 . Put

$$
\omega^{n}(x)=\omega\left(\omega^{n-1}(x)\right)=\Delta_{\alpha_{n+1}(x) \alpha_{n+2}(x) \ldots}^{G_{2}} .
$$

Since

$$
x=\delta_{\alpha_{1}(x)}+\sum_{k=2}^{n}\left(\delta_{\alpha_{k}(x)} \prod_{j=1}^{k-1} g_{\alpha_{j}(x)}\right)+\left(\prod_{j=1}^{n} g_{\alpha_{j}(x)}\right) \omega^{n}(x),
$$

we have

$$
\omega^{n}(x)=\frac{x}{P_{n}(x)}-\frac{1}{P_{n}(x)}\left(\delta_{\alpha_{1}(x)}+\sum_{k=2}^{n}\left(\delta_{\alpha_{k}(x)} \prod_{j=1}^{k-1} g_{\alpha_{j}(x)}\right)\right) .
$$

Theorem 4 ([10]). Function $\omega^{n}$ is well defined by equality

$$
\omega^{n}(x)=\omega^{n}\left(\Delta_{\alpha_{1}(x) \alpha_{2}(x) \ldots \alpha_{n}(x) \ldots}^{G_{2}}\right) \equiv \Delta_{\alpha_{n+1}(x) \alpha_{n+2}(x) \ldots}^{G_{2}},
$$

is analytically expressed in the form $\omega^{n}(x)=\frac{1}{P_{n}} x-\frac{B_{n}}{P_{n}}$, where $P_{n}=$ $\prod_{j=1}^{n} g_{\alpha_{j}(x)}$,

$B_{n}=\delta_{\alpha_{1}(x)}+\sum_{k=2}^{n}\left(\delta_{\alpha_{k}(x)} \prod_{j=1}^{k-1} g_{\alpha_{j}(x)}\right)$, is continuous on interval $\left[0, g_{0}\right]$ and linear on every cylinder of rank $n$.

Remark 2. In terms of dynamical systems, relation "has the same tail" can be defined in the following form:

$$
x=\Delta_{\alpha_{1} \alpha_{2} \ldots \alpha_{n}}^{G_{2}} \sim y=\Delta_{\beta_{1} \beta_{2} \ldots \beta_{n}}^{G_{2}} \Leftrightarrow O_{x} \cap O_{y} \neq \varnothing
$$

where $O_{u}=\left\{u, \omega^{1}(u), \omega^{2}(u), \ldots\right\}$ is an orbit of point $u$ under mapping $\omega$, and $[x \wedge y]=O_{x} \cap O_{y}$.

Definition 3. Function $\tau_{i}$ defined on $\left[0, g_{0}\right]$ by equality

$$
\tau_{i}(x)=\tau_{i}\left(\Delta_{\alpha_{1}(x) \alpha_{2}(x) \ldots \alpha_{n}(x) \ldots}^{G_{2}}\right)=\Delta_{i \alpha_{1}(x) \alpha_{2}(x) \ldots \alpha_{n}(x) \ldots}^{G_{2}},
$$

where $i \in\{0,1\}$, is called a right shift operator of $G_{2}$-representation of numbers with parameter $i$ (in the sequel, we just say "right shift operator").

Since $\tau_{i}\left(\Delta_{c_{1} c_{2} \ldots c_{m} 01(0)}^{G_{2}}\right)=\tau_{i}\left(\Delta_{c_{1} c_{2} \ldots c_{m} 11(0)}^{G_{2}}\right)$, we see that function $\tau_{i}$ is well defined. It is evident that the set of values of function $\tau_{i}$ is cylinder $\Delta_{i}^{G_{2}}$. In particular,

$$
\tau_{0}(0)=\tau\left(\Delta_{(0)}^{G_{2}}\right)=0, \tau_{1}(0)=\tau_{1}\left(\Delta_{(0)}^{G_{2}}\right)=\Delta_{1(0)}^{G_{2}}=g_{0} .
$$


Lemma 1. Function $\tau_{i}$ is continuous at any point of interval $\left[0, g_{0}\right]$ and analytically expressed in the form $\tau_{i}(x)=\delta_{i}+g_{i} x$.

Indeed, since

$$
\tau_{i}\left(\Delta_{\alpha_{1} \alpha_{2} \ldots \alpha_{n} \ldots}^{G_{2}}\right)=\Delta_{i \alpha_{1} \alpha_{2} \ldots \alpha_{n} \ldots}^{G_{2}}=\delta_{i}+g_{i} \Delta_{\alpha_{1} \alpha_{2} \ldots \alpha_{n} \ldots}^{G_{2}},
$$

we have $\tau_{i}(x)=\delta_{i}+g_{i} x$, i.e., $\tau_{0}(x)=g_{0} x, \tau_{1}(x)=g_{0}+g_{1} x$. So, it is evident that function $\tau_{i}$ is continuous on cylinders of rank 1 . From equality $\tau_{i}\left(\Delta_{c_{1} c_{2} \ldots c_{m} 01(0)}^{G_{2}}\right)=\tau_{i}\left(\Delta_{c_{1} c_{2} \ldots c_{m} 11(0)}^{G_{2}}\right)$ it follows that the function is continuous at $G_{2}$-binary points that are endpoints of cylinders.

Corollary 1. Function $\tau_{0}$ is increasing and function $\tau_{1}$ is decreasing. Moreover, $\tau_{0}\left(g_{0}\right)=\tau_{1}\left(g_{0}\right)$.

The following equalities are evident: $\omega\left(\delta_{i}(x)\right)=x$ and $\tau_{\alpha_{1}(x)}(\omega(x))=$ $x$.

Equation $\tau_{i}(x)=\omega(x)$ has two solutions: $x=\Delta_{(j i)}^{G_{2}}$, where $j \in\{0,1\}$. Equation $\tau_{i}(x)=\omega^{m}(x)$ has $2^{m}$ solutions: $x=\Delta_{\left(j_{1}, j_{2}, \ldots, j_{m} i\right)}^{G_{2}}$, where $j_{k} \in$ $\{0,1\}, k=\overline{1, m}$.

Let $\left(i_{1}, i_{2}, \ldots, i_{n}\right)$ be a tuple of zeros and ones. Function $\tau_{i_{1} i_{2} \ldots i_{n}}$ defined by equality $\tau_{i_{1} i_{2} \ldots i_{n}}(x)=\Delta_{i_{1} i_{2} \ldots i_{n} \alpha_{1}(x) \alpha_{2}(x) \ldots}^{G_{2}}$ is called a right shift operator with parameters $\left(i_{1}, i_{2}, \ldots, i_{n}\right)$. By induction, from equality $\tau_{i_{1} i_{2} \ldots i_{n}}(x)=$ $\tau_{i_{1}}\left(\tau_{i_{2} \ldots i_{n}}(x)\right)$ it follows that operator $\tau_{i_{1} i_{2} \ldots i_{n}}(x)$ is well defined.

Operator $\tau_{i_{1} i_{2} \ldots i_{n}}$ is analytically expressed in the form

$$
\tau_{i_{1} i_{2} \ldots i_{n}}(x)=\delta_{i_{1}}+\sum_{k=1}^{n}\left(\delta_{i_{k}} \prod_{j=1}^{k-1} g_{i_{j}}\right)+\left(\prod_{j=1}^{n} g_{i_{j}}\right) x,
$$

is a linear function, is increasing if $P_{n}=\prod_{j=1}^{n} g_{i_{j}}>0$ (this is equivalent to $i_{1}+i_{2}+\ldots+i_{n}$ is even number) and decreasing if $P_{n}<0$ (this is equivalent to $i_{1}+i_{2}+\ldots+i_{n}$ is odd number).

For example, consider $n=2$ and corresponding functions $\tau_{00}, \tau_{01}, \tau_{10}$, $\tau_{11}$. Functions $\tau_{00}=g_{0}^{2} x$ and $\tau_{11}=g_{0}^{2} x+g_{0}^{2}$ are linear increasing, but functions $\tau_{01}=g_{0} g_{1} x+g_{0}^{2}$ and $\tau_{10}=g_{0} g_{1} x+g_{0}$ are linear decreasing.

\section{Continuous functions and transformations of interval $\left[0, g_{0}\right]$ preserving tails of $G_{2}$-representation of numbers}

We say that function $y=f(x)$ preserves tails of $G_{2}$-representation of numbers in interval $\left[0, g_{0}\right]$ (or is a tail function) if any number $x \in\left[0, g_{0}\right]$ and its image $y=f(x)$ have the same tail. 
Left and right shift operators $\omega^{n}, \tau_{i_{1} i_{2} \ldots i_{n}}$ for any positive integer $n$ and for any tuple $\left(i_{1}, i_{2}, \ldots, i_{n}\right)$ of zeros and ones are simple examples of continuous functions preserving tails of $G_{2}$-representation of numbers. Various "joinings" of these functions are the same. For example, function

$$
f(x)= \begin{cases}\omega(x) & \text { if } 0 \leqslant x \leqslant x_{1} \\ \tau_{1}(x) & \text { if } x_{1} \leqslant x \leqslant x_{2} \\ \omega(x) & \text { if } x_{2} \leqslant x \leqslant g_{0}\end{cases}
$$

where $x_{1}$ and $x_{2}$ are solutions of equation $\omega(x)=\tau_{1}(x)$, i.e., $x_{1}=\Delta_{(01)}^{G_{2}}$, $x_{2}=\Delta_{(1)}^{G_{2}}$, preserves tails of $G_{2}$-representation.

But not every continuous function defined on interval $\left[0, g_{0}\right]$ is its transformation, i.e., bijection of the interval to itself. It is clear that above mentioned functions are not transformations.

It is clear that continuous transformations of interval $\left[0, g_{0}\right]$ can be only strictly monotonic (increasing and decreasing) functions such that their domain and set of values coincide with this interval.

Lemma 2. Decreasing function

$$
f_{1}(x)= \begin{cases}\tau_{1}(x) & \text { if } x \leqslant x_{1}=\Delta_{(1)}^{G_{2}}=\frac{g_{0}}{2-g_{0}}, \\ \omega(x) & \text { if } x \geqslant x_{1}=\Delta_{(1)}^{G_{2}}=\frac{g_{0}}{2-g_{0}},\end{cases}
$$

is a continuous tail transformation of interval $\left[0, g_{0}\right]$.

Proof. Number $x_{1}$ is a solution of equation $\tau_{1}(x)=\omega(x)$ being equivalent to system of equations $1=\alpha_{2}(x)=\alpha_{4}(x)=\ldots, \alpha_{1}(x)=\alpha_{3}(x)=$ $\alpha_{5}(x)=\ldots$ So this equation has two solutions: $x=\Delta_{\left(\alpha_{1} 1\right)}^{G_{2}}, \alpha_{1} \in\{0,1\}$. First solution $x_{0}=\Delta_{(01)}^{G_{2}}$ belongs to interval of decrease of function $\tau_{1}$ and to interval of increase of function $\omega$, and second solution $x_{1}$ belongs to interval of decrease of function $\tau_{1}$ and to interval of decrease of function $\omega$. Thus $f_{1}$ is a continuous and strictly decreasing function. Moreover, $f_{1}(0)=$ $g_{0}$ and $f_{1}\left(g_{0}\right)=0$. Hence $f_{1}$ is a continuous transformation of interval $\left[0, g_{0}\right]$.

Example 1. Function

$$
f_{2}(x)= \begin{cases}\tau_{1}(x) & \text { if } 0 \leqslant x \leqslant \Delta_{(101)}^{G_{2}}, \\ \omega^{2}(x) & \text { if } \Delta_{(101)}^{G_{2}} \leqslant x \leqslant g_{0},\end{cases}
$$

is a continuous decreasing tail transformation. 
Indeed, $\tau_{1}$ is a continuous decreasing tail function and $\Delta_{(101)}^{G_{2}}$ is a solution of equation $\tau_{1}(x)=\omega^{2}(x)$ belonging to the last interval of decrease of function $\omega^{2}(x)$.

Example 2. Decreasing function

$$
f_{3}(x)= \begin{cases}\tau_{1} \underbrace{0 \ldots 0}_{k}(x) & \text { if } 0 \leqslant x \leqslant x_{k} \equiv \Delta_{i 1}^{G_{2}} \underbrace{0 \ldots 0}_{k}, \\ \omega(x) & \text { if } x_{k} \leqslant x \leqslant g_{0}, i \in A,\end{cases}
$$

is a continuous tail transformation of interval $\left[0, g_{0}\right]$.

Indeed, $\omega$ and $\tau_{10 \ldots 0}$ are continuous tail functions, number $x_{k}$ is a solution of equation $\tau_{1} \underbrace{0 \ldots 0}_{k}(x)=\omega(x)$ belonging to intervals of decrease of both functions. Hence $f_{3}$ is a continuous decreasing tail function.

Theorem 5. The set $C$ of all continuous bijections of interval $\left[0, g_{0}\right]$ preserving tails of $G_{2}$-representation of numbers with respect to composition (superposition) o forms an infinite non-abelian group such that increasing functions form its non-trivial subgroup.

Proof. It is known that the set of all bijections of interval forms a group such that an identity transformation is its neutral element and an inverse transformation is its symmetric element. It is evident that composition of tail transformations is a tail transformation. The same is true for inverse transformation. Thus, by the subgroup test, $(C, \circ)$ is a group. From example 3 (where $k$ is an arbitrary positive integer) it follows that this group is infinite.

To prove that group $(C, \circ)$ is non-abelian it is enough to provide two transformations in the set $C$ that are not commute. To this end we consider function $f_{1}$ and $f_{2}$ from examples 1 and 2 and number $x_{0}$ that is less than $x_{1}$. Then we have

$$
\begin{aligned}
f_{2}\left(f_{1}\left(\Delta_{01(0)}^{G_{2}}\right)\right) & =f_{2}\left(\tau_{1}\left(\Delta_{01(0)}^{G_{2}}\right)\right)=f_{2}\left(\Delta_{101(0)}^{G_{2}}\right) \\
& =\tau_{1}\left(\Delta_{101(0)}^{G_{2}}\right)=\Delta_{1101(0)}^{G_{2}}
\end{aligned}
$$

because of $\Delta_{101(0)}^{G_{2}}<\Delta_{(101)}^{G_{2}}$;

$$
\begin{aligned}
f_{1}\left(f_{2}\left(\Delta_{01(0)}^{G_{2}}\right)\right) & =f_{1}\left(\tau_{1}\left(\Delta_{01(0)}^{G_{2}}\right)\right)=f_{1}\left(\Delta_{101(0)}^{G_{2}}\right) \\
& =\omega\left(\Delta_{101(0)}^{G_{2}}\right)=\Delta_{01(0)}^{G_{2}}
\end{aligned}
$$


because of $\Delta_{101(0)}^{G_{2}}>\Delta_{(1)}^{G_{2}}$. Hence $f_{2}\left(f_{1}\left(x_{0}\right)\right) \neq f_{1}\left(f_{2}\left(x_{0}\right)\right)$.

To prove that there exists a non-trivial subgroup of increasing functions it is enough to give an example of non-trivial increasing bijection $f \in C$. A such function is the following:

$$
f_{5}(x)= \begin{cases}\omega(x) & \text { if } 0 \leqslant x \leqslant \Delta_{(011)}^{G_{2}}, \\ \tau_{11}(x) & \text { if } \Delta_{(011)}^{G_{2}} \leqslant x \leqslant \Delta_{(1)}^{G_{2}}, \\ x & \text { if } \Delta_{(1)}^{G_{2}} \leqslant x \leqslant g_{0},\end{cases}
$$

because functions $\omega, \tau_{11}$ and $f(x)=x$ are increasing on given intervals, $\Delta_{(011)}^{G_{2}}$ is a solution of equation $\omega(x)=\tau_{11}(x)$, and $\Delta_{(1)}^{G_{2}}$ is a solution of equation $\tau_{11}(x)=x$.

Remark 3. The group $(C, \circ)$ is a proper subgroup of the group of transformations of interval $\left[0, g_{0}\right]$ preserving frequencies of digits of representation.

\section{References}

[1] M. Iosifescu, C. Kraaikamp, Metric properties of Denjoy's canonical continued fraction expansion, Tokyo J. Math., 31, no. 2, 2008, pp. 495-510.

[2] T. M. Isaieva, M. V. Pratsiovytyi, Transformations of $(0,1]$ preserving tails $\Delta^{\mu}$ representation of numbers, Algebra Discrete Math., 22, no. 1, 2016, pp. 102-115.

[3] M. Pratsiovytyi, A. Chuikov, Continuous distributions whose functions preserve tails of an A-continued fraction representation of numbers, Random Oper. Stoch. Equ., 27, no. 3, 2019, pp. 199-206.

[4] R. Yu. Osaulenko, Group of transformations of interval [0,1] preserving frequencies of digits of $Q_{s}$-representation of numbers, Trans. Inst. Math. Natl. Acad. Sci. Ukraine, 13, no. 3, 2016, pp. 191-204 (in Ukrainian).

[5] M. V. Pratsiovytyi, Random variables with independent $Q_{2}$-symbols, Asymptotic methods in investigation of stochastic models, Inst. Math. Acad. Sci. Ukrainian SSR, Kyiv, 1987, pp. 92-102 (in Russian).

[6] M. V. Pratsiovytyi, Fractal properties of distributions of random variables whose $Q_{2}$ signs form a homogeneous Markov chain, Asymptotic analysis of random evolutions, Inst. Math. Acad. Sci. Ukraine, Kyiv, 1994, pp. 249-254 (in Ukrainian).

[7] M. V. Pratsiovytyi, Yu. P. Maslova, On one generalization of system of Rademacher and Walsh functions, Mathematical problems of mechanics and computational mathematics. Trans. Inst. Math. Natl. Acad. Sci. Ukraine, 13, no. 3, 2016, pp. 146157 (in Ukrainian).

[8] M. V. Pratsiovytyi, I. M. Lysenko, Yu. P. Maslova, Geometry of numerical series: Series as a model of a real number in a new two-symbol system of encoding of numbers, Mathematical problems of mechanics and computational mathematics. Trans. Inst. Math. Natl. Acad. Sci. Ukraine, 15, no. 1, 2018, pp. 132-146 (in Ukrainian). 
[9] M. V. Pratsiovytyi, Fractal approach in investigation of singular probability distributions, Natl. Pedagog. Dragomanov Univ. Publ., Kyiv, 1998 (in Ukrainian).

[10] M. V. Pratsiovytyi, I. M. Lysenko, Yu. P. Maslova, Numeral system with two bases having different signs and related special functions, To appear in Mathematical problems of mechanics and computational mathematics. Trans. Inst. Math. Natl. Acad. Sci. Ukraine, 16, 2019 (in Ukrainian).

\section{CONTACT INFORMATION}

Mykola

V. Pratsiovytyi, Iryna M. Lysenko, Yuliya P. Maslova
Institute of Mathematics,

National Academy of Sciences of Ukraine, Tereschenkivska str. 3,

Institute of Physics and Mathematics,

National Pedagogical Mykhailo Drahomanov

University, 9 Pyrohova St., Kyiv, 01601, Ukraine

$$
\begin{aligned}
\text { E-Mail(s): } & \text { prats4444@gmail.com, } \\
& \text { iryna.pratsiovyta@gmail.com, } \\
& \text { julia0609mas@gmail.com }
\end{aligned}
$$

Received by the editors: 21.11.2019

and in final form 11.01.2020. 